\title{
Deutsche Akademie für Seltene Neurologische Erkrankungen
}

\section{Konzepte und Struktur}

\section{Schöls'; H. Graessner²; A. Münchau³}

${ }^{1}$ Sektion Klinische Neurogenetik, Neurologische Klinik und Hertie Institut für Klinische Hirnforschung, Universität Tübingen; ${ }^{2}$ Institut für Medizinische Genetik und angewandte Genomik, Universität Tübingen; ${ }^{3}$ Institut für Neurogenetik, Universität zu Lübeck, Universitätsklinikum Schleswig-Holstein, Campus Lübeck

\section{Schlüsselwörter}

Seltene neurologisches Krankheiten, Akademie, Fallkonferenz, Internet-Platform

\section{Zusammenfassung}

Die Deutsche Akademie für Seltene Neurologische Erkrankungen (DASNE) will Wegbereiter für eine optimierte Versorgung von $\mathrm{Pa}$ tienten mit seltenen neurologischen Erkrankungen sein. Als erste Institution in Deutschland verfolgt die Akademie die Bündelung und kontinuierliche Weiterentwicklung von Expertise im Bereich seltener neurologischer Erkrankungen durch den Zusammenschluss deutscher Expertenzentren. Ausgehend von den Initiatoren in Lübeck, Tübingen und Bonn haben sich weitere Zentren angeschlossen. Ein dynamisches Netzwerk beginnt sich zu entwickeln. In diesem Verbund decken DASNE-Experten die multidisziplinäre Expertise zu seltenen neurologischen Erkrankungen vom Kindes- bis zum Erwachsenenalter ab. Gemeinsame Falldiskussionen und zentrale Datenerfassung eröffnen neue Wege. Hauptziele der DASNE sind die Etablierung eines deutschen Expertise-Netzwerks für seltene neurologische Erkrankungen, der Aufbau einer Web-Plattform zur Erfassung von Patientendaten für videobasierte Fallkonferenzen mit Experten und moderierten Austausch zu seltenen neurologischen Erkrankungen sowie Aus- und Fortbildung junger in den Neurofächern tätiger Kollegen in Workshops zu zentralen Themen seltener neurologischer Erkrankungen.

\section{Keywords}

Rare neurological disorders, Academy, case conference, web based platform

\section{Summary}

The German Academy of Rare Neurological Disorders (Deutsche Akademie für Seltene Neurologische Erkrankungen; DASNE) aims to pave the way for an optimized management of patients with rare neurological disorders. By bringing together experts in the field in a dynamic national network the DASNE, initiated by the Centers for Rare Diseases in Lübeck, Tübingen and Bonn, will continuously foster mutual exchange. Members of the DASNE are nationally and internationally renowned experts covering the whole spectrum and age range of rare neurological disorders including pediatric neurology. Through case presentations and discussion both at yearly meetings and on an internet platform the DASNE's main aims are to establish a German expertise and reference network for rare neurological disorders, to set up a web based platform for case conferences including patient videos and imaging data and to offer continuous medical education for younger academics in the field of rare neurological disorders.

German Academy of rare neurological disorders Concepts and structure

Nervenheilkunde 2018; 37: 136-140

eingegangen am: 16. Oktober 2017

angenommen am: 1. Dezember 2017
Definitionsgemäß betreffen seltene Erkrankungen $<1 / 2000$ Menschen, sodass es für Ärzte außerhalb von spezialisierten Zentren schwer ist, für sich eigene Erfahrung und Expertise aufzubauen. Trotz der Seltenheit der einzelnen Erkrankung leiden Schätzungen zufolge in Deutschland etwa 4 Millionen Menschen an einer der rund 7000-8000 bekannten seltenen Erkrankungen (http://www.namse.de/aktionsbu endnis.html). Die Zahl der molekular definierten Erkrankungen nimmt dank der Fortschritte in der genetischen Forschung rasch zu. Bislang sind etwa 1000 neurologische seltene Erkrankungen definiert, unter anderem $>70$ Formen hereditärer sensomotorischer Neuropathien, $>25$ Gliedergürtelmuskeldystrophien, $>50$ mitochondriale Zytopathien, $>80$ Subtypen hereditärer spastischer Spinalparalysen, > 30 erbliche Leukenzephalopthien, > 50 Formen epileptischer Enzephalopathien, um nur einige wenige größere Gruppen zu nennen, des weiteren beispielsweise $>90$ Gene für hereditäre Ataxien und $>50$ Gene für nicht syndromale X-gebundene mentale Retardierung (https://www.omim.org/).

Erkenntnisse zu den zumeist genetischen Grundlagen zahlreicher neu definierter seltener neurologischer Erkrankungen und die sich hieraus ergebende überaus große Vielfalt zu bewertender genetischer Varianten und beständig neu zu bestimmender Genotyp-Phänotyp-Beziehungen nehmen gegenwärtig in rasantem Tempo zu. Dadurch werden an in den Neurofächern tätige Neurologen, Neuropädiater und Neurowissenschaftler in Hinblick auf Diagnostik und Therapie vieler dieser „neuen“ bzw. neu zu definierenden Erkrankungen große Anforderungen gestellt. Trotz großer diagnostischer Fortschritte 


\section{DASNE (1)}

lassen sich etwa 50\% seltener neurologischer Erkrankungen bzw. Syndrome nicht klar zuordnen, bzw. sind noch nicht verstanden. In Deutschland kann geschätzt von einer Prävalenz von 150000 Patienten mit seltenen neurologischen Erkrankungen und einer Inzidenz von 7000-8000 Neuerkrankungen pro Jahr ausgegangen werden. Angesichts der Seltenheit einzelner klinisch-neurogenetischer Szenarien und des notwendigen hohen Spezialisierungsgrads von Experten in verschiedenen Themenfeldern, kann eine den unterschiedlichen $\mathrm{Fa}$ cetten gerecht werdende Expertise von einem neurologischen Zentrum allein nicht gewährleistet werden. Zur Wahrung bzw. Entwicklung einer dem Stand des gegenwärtigen Wissens entsprechenden höchsten Qualität und volkswirtschaftlich möglichst optimalen Ressourcen-Zuordnung und -Nutzung bei diagnostischen Prozessen und der Behandlung seltener neurologischer Erkrankungen sind neue klinische Strukturen und Formate erforderlich.

\section{DASNE - Grundkonzept}

Der Initiative zur Gründung der Deutschen Akademie für Seltene Neurologische Erkrankungen (DASNE) liegt der Kerngedanke zugrunde, dass ein den Ansprüchen und Potenzialen der deutschen Neurologie angemessener Umgang mit seltenen und unklaren Erkrankungen einer Bündelung und kontinuierlichen Entwicklung von Expertise in einem Zusammenschluss deutscher Expertenzentren zu einem sich weiter entwickelnden Expertise-Netzwerk bedarf. Kernelemente der DASNE sind ein jährliches Treffen, ein Bord von Experten und die Homepage, die alle einer dynamischen Entwicklung unterliegen sollen. Die DASNE-Experten sollen dabei die multidisziplinäre Expertise zu seltenen neurologischen Erkrankungen in allen Altersgruppen abdecken ( Tab. 1). In der DASNE werden Falldiskussionen der Experten und Datensammlung in telemedizinischen, webbasierten Strukturen Hand in Hand mit regelmäßigen persönlichen Treffen realisiert werden. Ein zentrales Anliegen der DASNE ist die aktive Einbindung des Nachwuchses als Teilnehmer in Fall-Präsentationen und Diskussion sowie Werk-
Tab. 1 Das DASNE-Experten-Panel

\begin{tabular}{|c|c|c|}
\hline Experte & Schwerpunkte /Spezialexpertise & Ort \\
\hline Assmann, Birgit & Neurometabolische Erkrankungen & Heidelberg \\
\hline Balint, Bettina & $\begin{array}{l}\text { Seltene Erkrankungen bei Erwachsenen, Stiff- } \\
\text { person-Syndrome }\end{array}$ & London, Heidelberg \\
\hline Bültmann, Eva & Neuroradiologie, Schwerpunkt Kinder & Hannover \\
\hline Boltshauser, Eugen & Neuropädiatrie, seltene Syndrome & Zürich \\
\hline Brockmann, Knut & $\begin{array}{l}\text { Neuropädiatrie, paroxysmale Störungen, in- } \\
\text { fantile Zerebralparese }\end{array}$ & Göttingen \\
\hline Brüggemann, Norbert & $\begin{array}{l}\text { Neurogenetik, Dystonien, genetisch determi- } \\
\text { nierte Parkinson Syndrome }\end{array}$ & Lübeck \\
\hline Ceballos-Baumann, Andres & Atypische Parkinson-Syndrome & München \\
\hline Ganos, Christos & $\begin{array}{l}\text { Hyperkinetische Bewegungsstörungen und } \\
\text { seltene Syndrome }\end{array}$ & Hamburg, Berlin \\
\hline Gasser, Thomas & $\begin{array}{l}\text { Neurogenetik, genetisch determinierte Parkin- } \\
\text { son Syndrome }\end{array}$ & Tübingen \\
\hline Gillessen-Kaesbach, Gabriele & Seltene genetische Syndrome, Syndromologie & Lübeck \\
\hline Gaessner, Holm & SE-Netzwerke, Struktur, Organisation & Tübingen \\
\hline Klein, Christine & $\begin{array}{l}\text { Neurogenetik, Dystonien, genetisch determi- } \\
\text { nierte Parkinson Syndrome }\end{array}$ & Lübeck \\
\hline Klockgether, Thomas & ZSE-Struktur, Ataxien & Bonn \\
\hline Klopstock, Thomas & Mitochondriopathien & München \\
\hline Krägeloh-Mann, Inge & $\begin{array}{l}\text { Neuropädiatrie, Leukodystrophien, infantile } \\
\text { Zerebralparese }\end{array}$ & Tübingen \\
\hline Lerche, Holger & Epilepsien, paroxysmale Störungen & Tübingen \\
\hline Leypold, Frank & Seltene neuroimmunologische Erkrankungen & Kiel \\
\hline Lohmann, Katja & Neurogenetik, Molekulargenetik & Lübeck \\
\hline Münchau, Alexander & $\begin{array}{l}\text { Bewegungsstörungen bei Kindern, seltene } \\
\text { neurologische Syndrome, Neuropsychiatrie }\end{array}$ & Lübeck \\
\hline Münte, Thomas & Neuropsychologie, kognitive Neurologie & Lübeck \\
\hline Opladen, Thomas & Neurometabolische Erkrankungen & Heidelberg \\
\hline Paus, Sebastian & Dystonien & Bonn \\
\hline Rieß, Olaf & Genetik, Ataxien & Tübingen \\
\hline Schnitzler, Alfons & Parkinson Syndrome & Düsseldorf \\
\hline Schramm, Peter & Neuroradiologie & Lübeck \\
\hline Schöls, Ludger & Ataxien, HSP, Leukodystrophien, Neurogenetik & Tübingen \\
\hline Schüle, Rebecca & HSP, seltene Syndrome. Neurogenetik & Tübingen \\
\hline Stephani, Ulrich & Pädiatrische Epileptologie & Kiel \\
\hline Synofzik, Matthis & $\begin{array}{l}\text { Ataxien, früh auftretende Demenzen und Mo- } \\
\text { toneuronerkrankungen, komplexe Bewe- } \\
\text { gungsstörungen, Neurogenetik }\end{array}$ & Tübingen \\
\hline Weber, Yvonne & Epilepsien, paroxysmale Störungen & Tübingen \\
\hline Wieczorek, Dagmar & Seltene genetische Syndrome, Syndromologie & Düsseldorf \\
\hline Wolff, Markus & Epilepsien bei Kindern und Erwachsenen & Tübingen \\
\hline
\end{tabular}




\section{DASNE (1)}

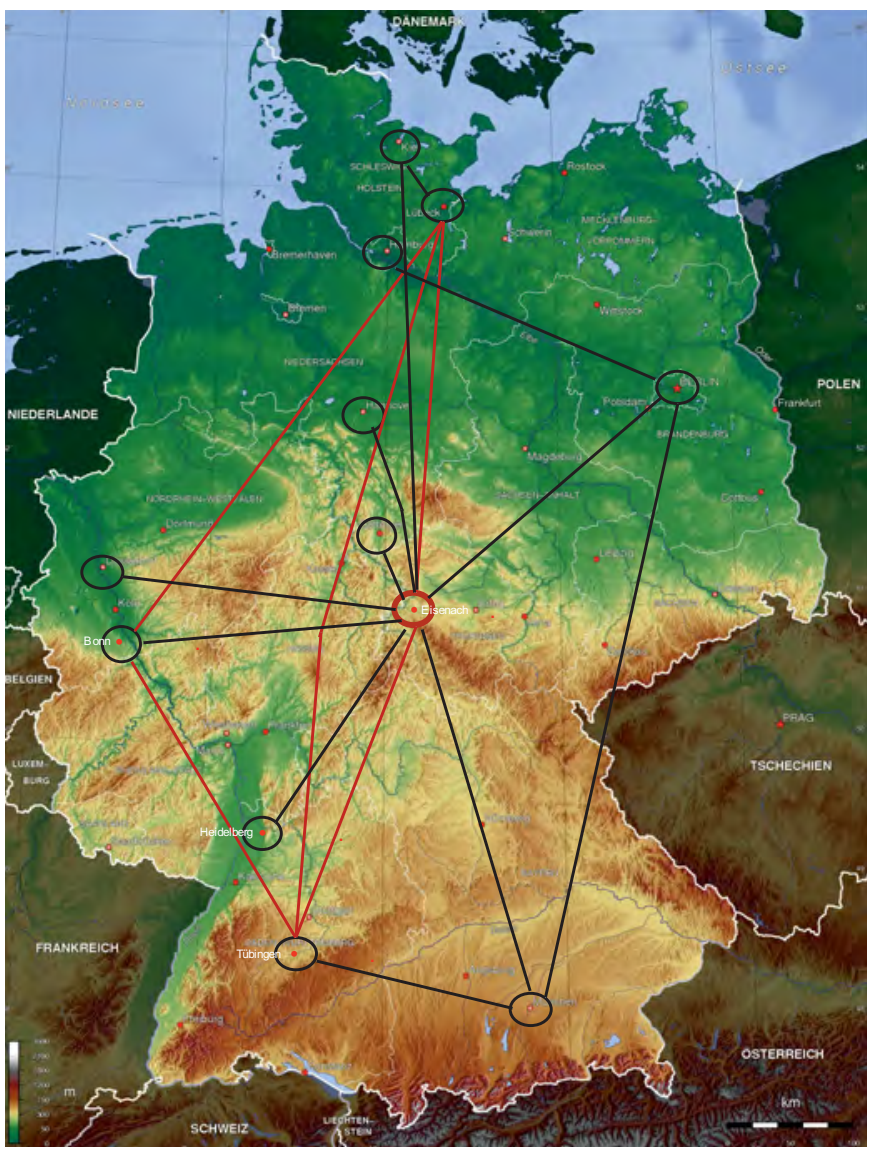

Abb. 1

DASNE-Experten-Netzwerk. Die Standorte der teilnehmenden deutschen Experten sind durch schwarze Kreise symbolisiert, bestehende enge Kooperationen im Feld der seltenen Erkrankungen durch schwarze Linien und die Verbindungen der Initiatoren der DASNE (Lübeck, Bonn und Tübingen) durch rote Linien. Rot umrandet ist der Ort des DASNE-Gründungssymposiums in Eisenach /auf der Wartburg als geografischer Mittelpunkt Deutschlands und ideengeschichtlicher Referenzort.

stätten zu Seltenen Erkrankungen ( $\triangleright$ Kasten). Die DASNE versteht sich als eine zu den an vielen Standorten in Deutschland entstandenen Zentren für Seltene Erkrankungen (ZSE) und den kürzlich durch die EU genehmigten Europäischen Referenznetzwerken (European Reference Networks; ERN) incl. des ERN on Rare Neurological Diseases (ERN-RND), des ERN on Neuromuscular Diseases (EURO-NMD) und des ERN on Epilepsies (EpiCARE) (1) komplementäre Institution, die exemplarisch als nationales Neuro-Expertise-Netzwerk in Deutschland eine Vorreiterrolle einnehmen soll ( $\triangleright$ Abb. 1).

\section{DASNE-Jahrestreffen}

Die Akademie soll mindestens einmal jährlich zwei Tage an einem zentralen Ort in Deutschland tagen. In dieser Zeit sollen lehrreiche geklärte und bislang ungelöste Fälle von einem Expertenforum ( $\triangleright$ Tab. 1) vorgestellt, diskutiert und für die Plattform aufgezeichnet werden. Patienten können in Videopräsentationen vorgestellt werden und sollen dann unter Einbeziehung der Teilnehmer differenzialdiagnostisch erörtert und in Hinblick auf die zugrunde liegende Pathophysiologie erläutert werden. Hierbei soll es wesentlich um einen engen Austausch klinisch und grundlagenwissenschaftlich tätiger Experten gehen. Die Jahrestreffen der Akademie knüpfen an bewährte Formate wie den „Syndromtag“ der Deutschen Gesellschaft für Humangenetik und den „Ungelösten Fall“ der Deutschen Gesellschaft für Neuropädiatrie an, erweitern diese jedoch wesentlich dadurch, dass der zuvor im Laufe des Jahres auf der Plattform fallbezogene Expertenaustausch in unterschiedlichen Präsentationsformen mit in die Diskussionen aufgenommen wird und der Vor-Ort-Austausch wiederum offline verfügbar gemacht wird. Durch die sich im Netzwerk- bzw. live entfaltende Fallbearbeitung sollen Rückschlüsse auf fruchtbare bzw. nicht zielführende Herangehensweisen gezogen werden können. Dabei soll nicht

\section{DASNE}

\section{Hauptziele}

Aufbau eines Expertise-Netzwerkes, einer Referenzressource und eines bundesweiten Registers für seltene neurologische Erkrankungen

Expertenaustausch zu klinischem, genetischem, immunologischem und molekularbiologischem Spezialwissen

Nachwuchsförderung

Optimierte Diagnosefindung und Therapie

Bewertung unklarer bzw. strittiger Fälle und Befunde (z. B. genetische Varianten)

die Einzelperformance, vielmehr die Kollektivbrillanz prägendes Gütekriterium sein.

\section{Gründungssymposium auf der Wartburg}

Das Gründungssymposium fand vom 29. November bis 1. Dezember 2017 auf der Wartburg und in Eisenach statt. Dieser Ort wurde bewusst gewählt, nicht nur als geografischer Mittelpunkt Deutschlands, vielmehr als ein Ort, der einen historischen Anknüpfungs- und Bezugspunkt für die DASNE darstellt. Am Hof des Landgrafen Hermann von Thüringen hatten sich nach etwas zweifelhafter Aktenlage, jedoch nachhaltiger Legendenbildung, 1207 erstmalig sechs Sänger auf der Wartburg versammelt, um in ihrer Kunst des Minnesangs $\mathrm{zu}$ brillieren und im Format des „Fürstenlobs“ im Wettstreit, im „Sängerkrieg", die Gunst des Herrschers und Ehre des Besten zu gewinnen (2). Dies waren Heinrich der tugendhafte Schreiber, Reinmar der Alte, Biterolf, Wolfram von Eschenbach, Walther von der Vogelweide und Heinrich von Ofterdingen. Dabei sollte der als schlechteste Bewertete, der Verlierer des Wettsingens, gemäß Vereinbarung an den Henker, Meister Stempfel, übergeben werden. Es traf Ofterding, der nicht den Landgrafen Hermann, sondern seinen eigenen Herrn, den Herzog von Österreich, besungen hatte. Durch Mitleid 


\section{DASNE (1)}

und Vermittlung der Ehefrau des Landgrafen, Sophie, wurde zur Schlichtung des potenziell fatalen - Wettstreits, der Gelehrte und Magier Klingsor aus Ungarn, selbst auch Dichter, gerufen, der dann im folgenden Jahr durch kluge Rede und etwas Magie den Konflikt schlichtete, den Sänger rettete und den Grundstein legte zu einem bemerkenswerten Format: dem „Rätsel-

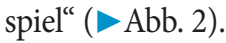

Klingsor gab Ofterding und in nachfolgenden Jahren anderen Sängern Rätsel auf, die sie lösen mussten. Es handelte sich um unklare Begebenheiten, Allegorien, „Fälle“. Die Zusammenkunft der Sänger unter Moderation des gelehrten Klingsor, dieser Sängerkrieg im Rätselspiel, galt einem ritterlichen Wissens- und Geisteskampf, einem Wettstreit der Fähigkeiten und Erfahrungen, und ausdrücklich nicht dem Gewinn einer Trophäe, einer Urkunde oder geldwertigen Belohnung. Ziel war die Erlangung der Meisterschaft, ein Ringen um der Sache selbst und um der Ehre willen. Dabei kam dem Wissen gleichermaßen eine Bedeutung zu wie dem Erkennen und Verstehen der Wissensquellen, um so die Macht des Unbekannten zu brechen. In den Konglomeraten von Strophengruppen der Sänger stehen die Ergebnisse, der Ausgang weniger im Vordergrund als der Prozess der Lösung und der Bewährung. Dem "Sängerkrieg" lag die Idee eines sich beständig miteinander messenden und vergleichenden kunstvoll singenden und durch die Kunst verbundenen Kollektivs, das Rätsel löst, zugrunde. Die Suche nach Erkenntnis und Verstehen war in seiner Konstruktion so verfasst, dass das tragende Hauptgerüst der Meisterschaft, der Expertise, in sich Öffnungen des Unwissens zuließ und sich damit auch an den noch nicht Wissenden, aber Interessierten, den inspirierten Zuhörer und Lernenden wendete. An diese Verfasstheit, an dieses Vorbild für die Beziehung zwischen Experten und Teilnehmern sucht die DASNE anzuknüpfen. Das Rätselspiel der Wartburg, begründet vor etwas mehr als 800 Jahren, das Sänger und Zuhörer faszinierte und sich in das Gedächtnis Nachfolgender einschrieb als eines der Ur-Ereignisse kollektiver Meisterschaft, die scheinbar unentwirrbare Rätsel $\mathrm{zu}$ lösen vermag, soll Leitbild und Motiv der DASNE sein.

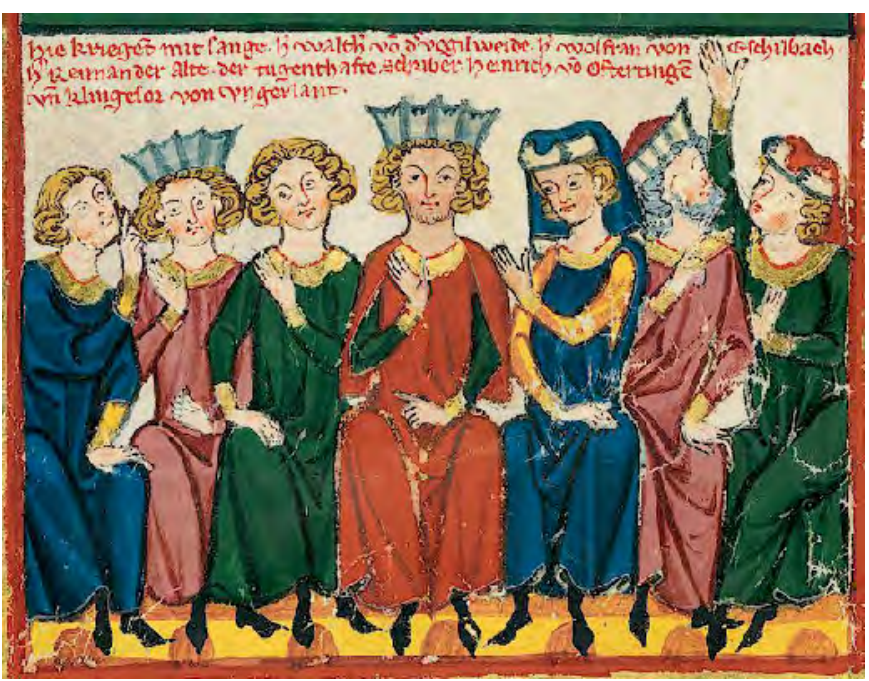

Abb. 2 Bildliche Darstellung des Sängerwettstreits auf der Wartburg. Es handelt sich um eine Miniatur aus dem Codex Manesse, einer mittelalterlichen Handschrift, die auch 137 ganzseitige farbige Miniaturen enthält. Die gezeigte Abbildung ist der untere Teil einer Miniatur (Tafel 72), die mittig Klingsor von Ungarland und zu seinen Seiten Walther von der Vogelweide, Wolfram von Eschenbach, Reinmar den Alten, Heinrich von Ofterdingen, den Tugenhaften Schreiber und Biterolf zeigt (aus Codex Manesse (3)).

\section{Aus- und Fortbildung}

In die Aktivitäten der DASNE sollen von Beginn an interessierte Kollegen eingebunden werden, um eine neue Neurologenge-

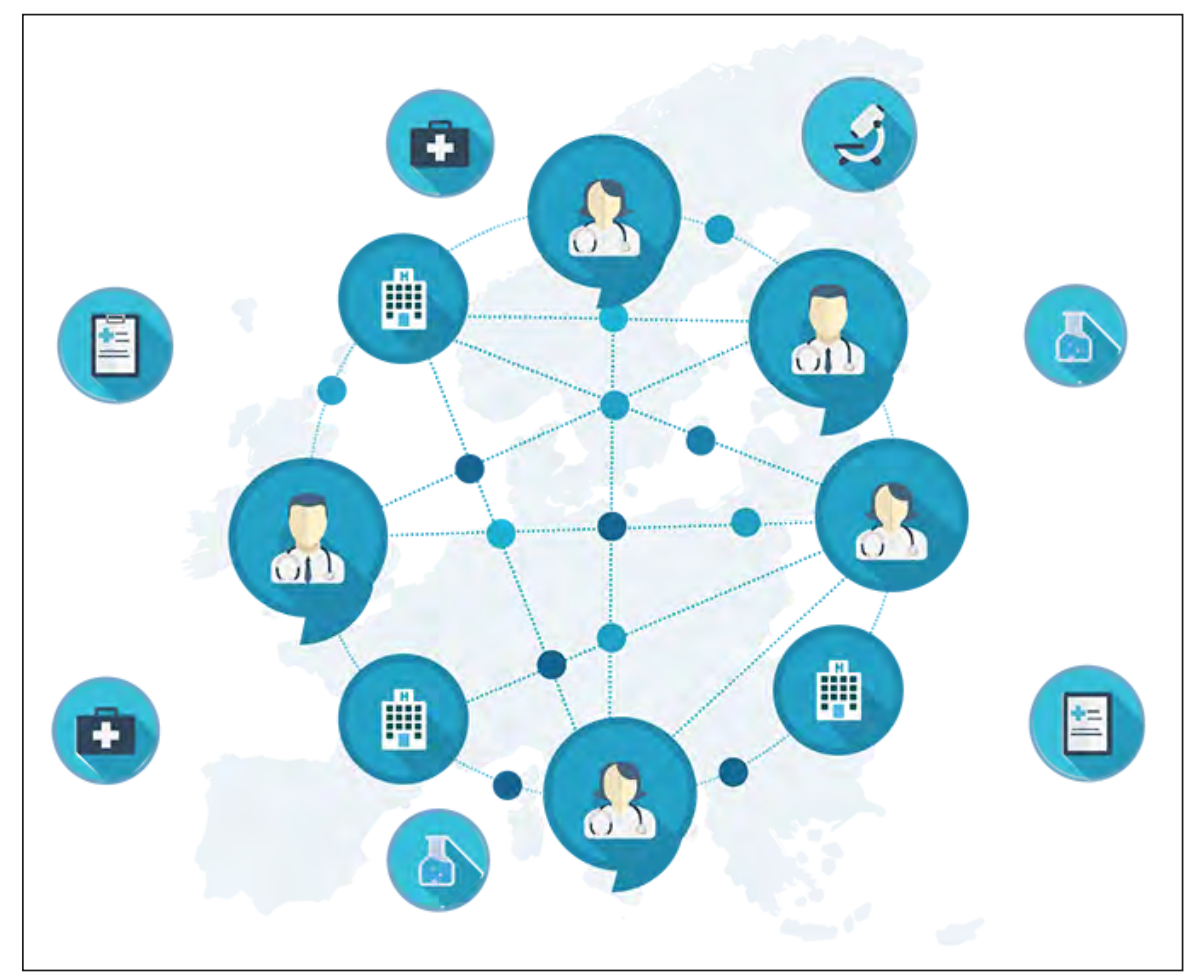

Abb. 3 Piktografische Darstellung des europäischen Versorgungsnetzwerkes, das durch die InternetPlattform aufgebaut werden wird. Quelle: Europäische Kommission. neration aus- bzw. fortbilden zu können. Diesem Ziel dienen sowohl die DASNEPlattform als auch die Jahrestreffen. Die DASNE-Plattform wird einen Fundus an Falldiskussionen generieren, die als Res- 
source für Aus- und Fortbildung genutzt werden können. Werkstätten (zu klinischer und radiologischer Mustererkennung, Syndromologie, Techniken des next generation sequencing sowie Interpretation genetischer Befunde) werden zusätzlich ein wesentlicher Bestandteil jedes Jahrestreffens sein.

\section{Internet-Plattform}

Es wird eine webbasierte Plattform genutzt, in der Patientendaten pseudonymisiert nach einer vorgegebenen Datenstruktur eingegeben und gespeichert werden ( $\triangle$ Abb. 3). Diese umfassen Angaben zu einer standardisierten klinischen Untersuchung, die Phänomenologie insbesondere von Bewegungsstörungen dokumentierende Videos und Ergebnisse von Zusatzuntersuchungen incl. der originalen Bildgebung (Dicom-Format) sowie genetisch-diagnostische Daten und die entsprechenden Stammbäume. Auf der Plattform erfolgen

- regelmäßige webbasierte Video-Fallkonferenzen unter Hinzuschalten eines oder mehrerer Experten und

- moderierte virtuelle Konsultationen, bei denen die Experten offline an Falldiskussionen teilnehmen können.

Die DASNE strebt an, die Falldiskussionsplattform zu nutzen, die auch von den Europäischen Referenznetzwerken verwendet werden wird. Diese Plattform wird gegenwärtig getestet und im Frühjahr 2018 nutzbar gemacht werden."

Ärztliche Kollegen, die für einen Patienten eine Konsultation erbitten, geben nach $\mathrm{Zu}$ - stimmung des Patienten gemäß einer vorgegebenen Datenstruktur Patientendaten auf der Plattform ein. Bei Annahme des Falles wird für jeden Patienten durch den verantwortlichen Moderator ein passendes Expertenpanel zusammengestellt, das den Fall diskutiert. In Anbetracht der im Augenblick $\mathrm{zu}$ erwartenden 50\%igen Lösungsquote ist es wichtig, dass der Workflow für jeden Fall in jedem Schritt neu begonnen werden kann. Das bedeutet zum Beispiel, dass für die Neudiskussion eines ungelösten Falles ein neues oder erweitertes Panel zusammengestellt werden kann, das zusätzliche nationale oder internationale Experten beinhalten kann.

Diskutierte Fälle werden anderen registrierten Teilnehmern der Plattform zur Verfügung gestellt. Dabei werden spezielle Rubriken zu definierten gelösten und zu unklaren Fällen eingerichtet. Zusätzlich kann auf der Plattform nach speziellen Fäl-

\section{Fazit für die Praxis}

Rasant wachsende Kenntnisse zu seltenen Erkrankungen erfordern neue Diskurs- und Ausbildungsformate. Dies strebt die DASNE in Gestalt eines interdisziplinären und altersübergreifenden Austauschs auf nationaler Ebene durch Kombination aus persönlichen Treffen und dauerhafter telemedizinischer Expertenplattform an. Die Hauptziele der DASNE sind eine verbesserte Diagnostik, individuell zugeschnittene Behandlungsstrategien, die Etablierung eines Referenznetzwerks für seltene neurologische Krankheiten und die Nachwuchsförderung. len beispielsweise mit definierter Ätiologie gesucht werden, was insbesondere in Hinsicht auf den über die Jahre wachsenden Fundus der Fälle bedeutsam ist. In Ergänzung zu den Fallkonferenzen bzw. virtuellen Konsultationen können zusätzlich Webinare zu relevanten Themen wie aktuellen Entwicklungen bei klinischen Studien organisiert werden. Der Zugang zur Plattform wird über die DASNE-Homepage möglich sein. Die DASNE-Homepage wird zusätzlich zu Informationen zur DASNE und dem Zugang zur Plattform online Rubriken z. B. zu kontroversen Themen und aktuellen Entwicklungen, enthalten. Die DASNE wird sowohl aufgrund der involvierten Experten als auch der akkumulierten Fallexpertise zusätzlich als Kristallisationspunkt und Informationsquelle für neue Register zu seltenen Erkrankungen und für neue Studien (inklusive Studienaufrufe) zu definierten seltenen Erkrankungen dienen.

\section{Interessenkonflikt}

Es bestehen keine Interessenkonflikte.

\section{Literatur}

1. Graessner H, Schäfer F, Scarpa M, Wagner TOF. European Reference Networks: Consequences for healthcare in Germany. Bundesgesundheitsblatt Gesundheitsforschung Gesundheitsschutz 2017; 60: 537-541.

2. Wachinger B. Sängerkrieg. Untersuchungen zur Spruchdichtung des 13. Jahrhundert. München: Verlag C.H. Beck 1973.

3. Codex Manesse. Die Miniaturen der Großen Heidelberger Liederhandschrift. Walther IF, Siebert G (Hrsg). Frankfurt am Main: Insel 2001. 


\title{
Zertifizierte Fortbildung
}

\section{in Zusammenarbeit mit der Bayerischen Landesärztekammer}

\author{
Fragen zum Thema „Deutsche Akademie für Seltene Neurologische \\ Erkrankungen"
}

1. Eine Erkrankung wird als selten definiert, wenn

a) weniger als 10000 Menschen weltweit betroffen sind.

b) weniger als 100000 Menschen weltweit betroffen sind.

c) die Häufigkeit $\leq 2 / 10000$ beträgt.

d) die Häufigkeit $\leq 1 / 2000$ beträgt.

e) die Häufigkeit $\leq 1 / 100000$ beträgt.

2. Welche der folgenden Erkrankungen ist nicht selten?

a) Friedreich Ataxie

b) Gilles-de-la-Tourette-Syndrom

c) Hereditäre Spastische Spinalparalyse

d) Morbus Huntington

e) Myotone Dystrophie

3. Welche der folgenden Aussagen trifft nicht zu?

a) Es sind über 70 Formen hereditärer sensomotorischer Neuropathien bekannt.

b) Derzeit sind > 25 Formen einer Gliedergürtelmuskeldystrophie charakterisiert.

c) Es gibt etwa 20 Subtypen mitochondrialer Zytopathien.

d) Über 30 Formen erblicher Leukenzephalopthien sind bekannt.

e) Die Gruppe epileptischer Enzephalopathien umfasst mehr als 50 Formen.

4. Wie hoch ist geschätzt die Prävalenz seltener neurologischer Erkrankungen in Deutschland?
a) 50000
b) 150000
c) 300000
d) 500000
e) 1000000

5. Welches der angeführten Netzwerke gehört nicht zu den von der EU anerkannten Europäischen Referenznetzwerke (ERN)?

a) Das ERN on Rare Neurological Diseases (ERN-RND).

b) Das ERN on Neuromuscular Diseases (EURO-NMD).

c) Das ERN on Neuro-Economics (Neuro-Euro).

d) Das ERN on Epilepsies (EpiCARE).

e) Das ERN on Congenital Malformations and Rare Intellectual Disability (ERN ITHACA).

6. Welche Aussage zur hereditären spastischen Spinalparalyse ist nicht korrekt?

a) Manche HSP Formen werden autosomalrezessiv vererbt.

b) Manche HSP Formen werden autosomaldominant vererbt.

c) Manche HSP Formen werden X-chromosomal vererbt.

d) Die Häufigkeit der meisten einzelnen Formen beträgt 2-10/100000.

e) Es sind über 80 genetische Unterformen bekannt.

7. Welches der folgenden Ziele ist nicht Hauptanliegen der „Deutsche Akademie für Seltene Neurologische Erkrankungen" (DASNE)?

a) Aufbau eines Expertise-Netzwerkes zur Diagnosefindung und Therapieplanung.

b) Aufbau von Registern für seltene neurologische Erkrankungen.

c) Persönlicher Expertenaustausch zu neurologischem Spezialwissen.

d) Nachwuchsförderung.

e) Leitlinienentwicklung.
Die Teilnahme an der CME-Fortbildung ist für 12 Monate ausschließlich online möglich. Zur Anmeldung gehen Sie bitte auf cme.schattauer.de. Dort erfahren Sie auch den genauen Teilnahmeschluss. Es ist immer nur eine Antwort pro Frage zutreffend. Als Leser der Nervenheilkunde nehmen Sie kostenlos am CME-Programm teil. Je nach CME-Fortbildung erhalten Sie bis zu vier Fortbildungspunkte. Weitere Informationen zur Anmeldung und Registrierung finden Sie unter cme.schattauer.de. Sie erhalten bei inhaltlichen und technischen Fragen tutoriellen Support.

8. Wie hoch ist in etwa die Wahrscheinlichkeit, durch den Einsatz moderner diagnostischer Verfahren incl. des Next Generation Sequencing und Expertenaustausch bei unklaren Erkrankung eine eindeutige Diagnose zu finden?
a) $5 \%$
b) $10 \%$
c) $30 \%$
d) $50 \%$
e) $80 \%$

9. Welche der folgenden Aussagen der Internetplattform der Deutschen Akademie für Seltene Neurologische Erkrankungen (DASNE) trifft nicht zu?

a) Die Plattform enthält Daten zur Phänomenologie incl. Patientenvideos.

b) Auf der Plattform werden Bildgebungsdaten zur Verfügung gestellt.

c) Auf der Plattform erfolgen webbasierte Video-Fallkonferenzen.

d) Fälle werden von einem festen Expertenpanel diskutiert.

e) Die Plattform ist in Europäische Referenznetzwerke eingebunden.

10. Welche der folgende Minnesänger nahm nicht am Sängerkrieg auf der Wartburg teil?

a) Heinrich der tugendhafte Schreiber

b) Reinmar der Alte

c) Der Wilde Alexander

d) Biterolf

e) Wolfram von Eschenbach 